Archives

$11 \mid 1993$

Histoire intellectuelle // Réflexions collectives sur l'histoire sociale

\title{
Le droit de bourgeoisie dans l'Europe moderne
}

Groupe de travail international. Paris, 7-8-9 octobre 1993

Simona Cerutti, Robert Descimon et Maarten Prak

\section{(2) OpenEdition}

\section{Journals}

Édition électronique

URL : http://journals.openedition.org/ccrh/2778

DOI : $10.4000 /$ ccrh. 2778

ISSN : $1760-7906$

Éditeur

Centre de recherches historiques - EHESS

Édition imprimée

Date de publication : 10 octobre 1993

ISSN : 0990-9141

Référence électronique

Simona Cerutti, Robert Descimon et Maarten Prak, «Le droit de bourgeoisie dans I'Europe moderne », Les Cahiers du Centre de Recherches Historiques [En ligne], 11 | 1993, mis en ligne le 05 mars 2009, consulté le 20 avril 2019. URL : http://journals.openedition.org/ccrh/2778 ; DOI : 10.4000/ccrh.2778

Ce document a été généré automatiquement le 20 avril 2019.

Article L.111-1 du Code de la propriété intellectuelle. 


\title{
Le droit de bourgeoisie dans l'Europe moderne
}

Groupe de travail international. Paris, 7-8-9 octobre 1993

\author{
Simona Cerutti, Robert Descimon et Maarten Prak
}

1 Comment penser aujourd'hui la nature de droits liés à une appartenance locale? Dans notre expérience quotidienne comme dans notre pratique d'historien(ne), les droits, prérogatives et privilèges, sont liés à des individus et à des groupes sociaux, à des personnes physiques ou fictives, non à des lieux, non à des pratiques sociales liées à des lieux.

2 Et pourtant, durant l'Ancien Régime, les villes d'Europe occidentale ont fait vivre le privilège de bourgeoisie au travers de modes de fonctionnement complexes qui reposaient moins sur une codification des normes que sur des pratiques accomplies par des personnes différentes dans des situations particulières de leur vie. Ce serait sans doute une erreur que de définir la «bourgeoisie » comme une «substance » sociale indifférenciée, ou dont les différences internes renverraient à un ailleurs, par exemple aux niveaux de fortune et aux distinctions de statuts. L'idée de hiérarchie est inhérente à la notion de bourgeoisie d'ancien régime. Dès lors, on se trouve confronté à la questionclef de l'articulation entre un lieu et une commuauté, entre des lieux, car toute ville forme une entité plurielle, et des communautés, car la bourgeoisie est une communauté qui réunit d'autres communautés.

3 On connaît les critères juridiques qui déterminent en général l'accès à la bourgeoisie : la naissance dans la cité ; la résidence, au moins lors des fêtes principales; l'hérédité du statut de bourgeois; le paiement d'un droit d'entrée; la participation aux charges communes; l'inscription sur un livre... La constitution éventuelle d'un patriciat qui monopolise plus ou moins le magistrat urbain, la différenciation officielle des bourgeois en plusieurs classes, la distinction entre «bourgeois ", «manants » et " habitants ", viennent souvent sanctionner des clivages internes que peuvent rendre encore plus complexes les oppositions religieuses, l'appartenance confessionnelle étant elle-même 
parfois érigée en critère discriminant pour l'obtention du droit de bourgeoisie. Ces aspects peuvent être approfondis à la lumière d'une approche comparative.

Mais l'idée de communauté sous-jacente aux pratiques bourgeoises renvoie à des stratifications plus subtiles reposant sur les capacites différentielles de certains groupes (lignages, jurandes, réseaux de clientèles, classes d'âge et de sexe...) à mettre en oeuvre le droit commun.

Deux convictions nous ont aidés à élaborer le présent projet avec une sorte de fidélité à la nature même de l'objet. Nous pensons que les droits liés à la bourgeoisie sont mis en oeuvre dans des situations concrètes à l'interieur de rapports de conflits avec d'autres systèmes de classification sociale. Nous pensons aussi qu'une pluralité de systèmes normatifs, différents et parfois contradictoires, caractérisent la vie urbaine.

La ville et la bourgeoisie se trouvèrent soumis à la concurrence de formations politiques et sociales rivales, qu'on désigne généralement sous le nom d'« État » et de «noblesse de fonction ». Une réflexion sur les rapports entretenus par les deux systèmes, sur leur genèse respective et leur engendrement réciproque, constitue la toile de fond de notre projet. La construction des États territoriaux a imposé aux communautés bourgeoises certains rythmes et processus de mutation : grossièrement, on peut repérer un effort de classification de la population pour faire prévaloir les conditions de continuité essentielles au prélèvement et à la police. Les pouvoirs urbains s'exercent souvent comme s'emboîtent des poupées russes. Les bourgeoisies se définissent aussi sans doute par leur capacité d'« ubiquité », dans la « ville » bien sûr, mais aussi dans « l'État » : la multiplicité des positions et des jeux de pouvoirs crée et révèle les stratifications internes de la communauté.

Quatre champs d'analyse nous semblent introduire à la compréhension des mécanismes de délimitation et de redistribution qui fondent les pratiques bourgeoises.

8 1. Le premier de ces champs s'identifierait aux implications sociales de la fiscalité urbaine : dialectique des « deniers communs » et des « dons gratuits » (pour reprendre la terminologie française); dialectique des «droits d'entrée» et des « capitations»; dialectique de l'emprunt, forcé ou non, de la rente et de la fiscalité... Jusqu'à quel point le modèle de l'imposition municipale, souvent hostile à l'enregistrement des biens et à la constitution de listes de contribuables, s'efforçait-il de concilier la connaissance des niveaux de prestige et donc de fortune, avec la reconnaissance des principes d'autorité liés à la résidence?

9 2. Le second champ nous entraînerait du côté du droit à l'assistance. Les critères de sélection mis en oeuvre par les groupes dirigeants qui contrôlaient les institutions de charité ne correspondaient pas à des niveaux de pauvreté, ni non plus à des réseaux de clientèles. Ces critères exprimaient des hiérarchies de légitimité à l'égard de la protection sociale. Comment s'articulaient-ils avec les différences de richesse, de sexe, d'âge et de profession?

10 3. Le troisième champ conduirait à nous intéresser aux pratiques militaires ou quasi militaires qui, dans beaucoup de cités, ont amené l'établissement de milice ou de guet bourgeois. La participation à ces services de garde aux portes, aux murailles ou dans le quartier, constituait une lourde charge, mais aussi un honneur et un signe d'intégration à la communauté, même s'il n'y a pas toujours identité entre le droit de bourgeoisie et le service militaire. L'organisation de la milice par métiers ou par quartiers, permet d'autre 
part de dégager des modèles d'organisation du privilège de citoyenneté, souvent aussi en liaison avec les modes de désignation du magistrat urbain.

11 4. Le quatrième champ coïnciderait avec les prérogatives juridictionnelles attribuées aux municipalités et les privilèges de juridiction attribués aux bourgeois : la ville d'Ancien Régime abrite une pluralité de systèmes juridiques, dont chacun s'appuie sur une conception différente de la personne légale, du statut de la preuve, du statut du serment, du statut du domicile... En outre, la communauté anime un système d'« infra-justice » très actif où les normes s'expriment au-delà du droit positif pour fonder en permanence le lien social, sans recours au politique mais dans la reconnaissance d'un pur pouvoir social. Le droit de bourgeoisie fonctionne-t-il en tant que critère fondamental de distinction au sein de ces différents champs? Comment s'articule-t-il avec les autres éléments de la hiérarchie sociale ? Comment un droit lié à la localité peut-il montrer de l'efficacité dans un système global qui vise à affirmer son caractère supra-local ?

Il nous faudrait aussi prendre en compte l'hétérogéneité de l'espace urbain: villes et faubourgs, cités et bourgs, multiplicité des seigneuries et des justices urbaines (créant des dénivellations dans l'espace économique et la vie des métiers), diversité des paroisses et des quartiers eux-mêmes...

14 Les niveaux d'analyse sont donc complexes, mais nous les croyons cohérents : les études urbaines abordent souvent ces thèmes dans un même cadre. Le projet vise moins à donner une synthèse conceptuelle du droit de bourgeoisie qu'à réussir une incursion dans des champs disparates, souvent déjà défrichés, mais qui trouveraient une fertilité nouvelle à cette interrogation spécifique : comment fonctionnait concrètement, dans les villes d'Ancien Régime, un principe de stratification urbaine tel que le droit de bourgeoisie?

\section{PROGRAMME}

Simona CERUTTI (EHESS) LE STATUT JURIDIQUE DE L'IDENTITÉ URBAINE (TURIN XVII ${ }^{\mathrm{e}}-\mathrm{XVIII}^{\mathrm{e}}$ SIÈCLE) : MARCHANDS ET DROIT DE CITÉ.

Maarten PRAK (Utrecht), THE RIGHT OF CITIZENSHIP IN DUTCH CITIES UNTIL THE ESTABLISHMENT OF THE KINGDOM OF THE NETHERLANDS (1813) : A COMPARATIVE ANALYSIS.

Jonathan BARRY (EXeter),MEANING OF FREEDOM : THE CHANGING SIGNIFICANCE OF FREEDOM IN EARLY MODERN ENGLAND.

Neithard BULST (Bielefeld),LES APPARENCES DE LA BOURGEOISIE (ALLEMAGNE ET FRANCE - XV ${ }^{\mathbf{e}}$ SIÈCLE).

GuY LEMEUNIER (CNRS),ETRE VECINO À MURCIE (VERS 1480 - VERS 1620).

Anna BELLAVITIS (EHESS), LA CITOYENNETÉ À VENISE AU XVI ${ }^{\mathrm{e}}$ SIÈCLE.

Regula SCHMID (Zurich), THE PRACTICES OF CITIZENSHIP RIGHTS IN BERN AND ZURICH (VERS. 1450 - VERS. 1550).

Ian W. ARCHER (Oxford), CITIZENSHIP IN LONDON (VERS 1450 - 1700).

R. Po-chia Hsia (New York), CITIZENSHIP AND MINORITY RIGHTS : JEWS AND BÜRGER IN THE EARLY MODERN GERMAN CITY.

Jacques Revel (EHESS),LES JUIFS ET LA CONQUÊTE DE LA CITOYENNETÉ FRANÇAISE (XVIII ${ }^{\mathrm{e}}$ SIÈCLE). Peter SAHLINS (Berkeley), LE DROIT D'AUbAINE ET LES PRATIQUES DE LA NATIONALité EN FRANCE AUX XVII ${ }^{\mathrm{e}}$ ET XVIII ${ }^{\mathrm{e}}$ SIÈCLES. 
27 Piero VENTURA (Sapienza-Rome), FRONTIÈRES FAIBLES OU ESPACE FONCTIONNEL ? POUVOIR POLITIQUE, GROUPES SOCIAUX ET PRIVILÈGES DE CITOYENNETÉ NAPOLITAINE ENTRE XVI ${ }^{\mathrm{e}}$ ET XVII ${ }^{\mathrm{e}}$ SIÈCLES

28 Gerald SOLIDAY (Dallas), MUNICIPAL PRIVILEGE AND STATE CONSCIOUSNESS : CITIZENSHIP IN EARLY MODERN GERMAN CITIES.

29 Robert DESCIMON (EHESS),LE CORPS DES BOURGEOIS ET LES CORPS DE MÉTIER À PARIS (MI-XV - MI-XVII ${ }^{\mathrm{e}}$ SIÈCLE).

30 Marc BOONE (Ghent), PARTICULARISME URBAIN ET DROIT DE BOURGEOISIE DANS LA FLANDRE BOURGUIGNONNE ET HABSBOURGEOISE (1384-1585).

31 Cesare MOZZARELLI (Milan), QUELQUES HYPOTHÈSES AUTOUR DU DROIT DE CITÉ : ENTRE L'ORDRE COMMUNAL ET LE SYSTÈME PATRICIEN (ITALIE DU NORD ET CENTRALE, XV $\mathrm{XV}^{\mathrm{e}}-\mathrm{XVIII}^{\mathrm{e}} \mathrm{SIÈCLE}$ ).

32 William BEIK (Atlanta), LES ÉLITES MUNICIPALES FACE À L'ORDRE SOCIAL EN FRANCE AU XVII SIÈCLE. 